\title{
A proposal for a new classification of coracobrachialis muscle morphology
}

\author{
Bartłomiej Szewczyk $^{1} \cdot$ Michał Polguj $^{2} \cdot$ Friedrich Paulsen ${ }^{4,5} \cdot$ Michał Podgórski $^{3} \cdot$ Fabrice Duparc $^{6} \cdot$ Piotr Karauda $^{1}$. \\ Łukasz Olewnik ${ }^{1}$ [1]
}

Received: 13 November 2020 / Accepted: 27 January 2021 / Published online: 9 February 2021

(c) The Author(s) 2021

\begin{abstract}
Introduction The coracobrachialis muscle (CRM) originates from the apex of the coracoid process, in common with the short head of the biceps brachii muscle, and from the intermuscular septum. It inserts to the medial part of the humerus between the attachment of the medial head of the triceps brachii and the brachial muscle. Both the proximal and distal attachments of the CRM, as well as its relationship with the musculocutaneus nerve, demonstrate morphological variability.

Material and methods One hundred and one upper limbs (52 left, and 49 right) fixed in 10\% formalin solution were examined. Results Three main types, with subtypes, were identified. The most common was Type I (49.5), characterized by a single muscle belly with a classical origin from the coracoid process, medially and posteriorly to the tendon of the biceps brachii. Type II (42.6\%), characterized by two heads, was divided into two subtypes (A-B) depending on its origin: Type IIA, where one head originated from the coracoid process posteriorly to the tendon of the biceps brachii and the second head from the short head of the biceps brachii, and Type IIB, in which both heads originated from the coracoid process; however, the superficial head fused with the insertion of a short head of the biceps brachii, while the deep head was directly originating. Finally, Type III (7.9\%) was characterized by three heads: two originated from the coracoid process (superficial and deep), and the third from a short head of the biceps brachii. Two types of insertion and two types of musculocutaneous nerve (MCN) relative to CRM could be distinguished.

Conclusion An adapted classification is needed for all clinicians working in this area, as well as for anatomists. The CRM demonstrates morphological variability in both its proximal and distal attachments, as well as the variable course of the MCN relative to the CRM.

What is known about this subject "and" What this study adds to existing knowledge Not much is known about the variability of coracobrachialis muscle. The present paper introduces a completely new classification, both clinical and anatomical.
\end{abstract}

Keywords Coracobrachialis muscle $\cdot$ Coracobrachialis muscle $\cdot$ Musculocutaneous nerve $\cdot$ Median nerve $\cdot$ New classification

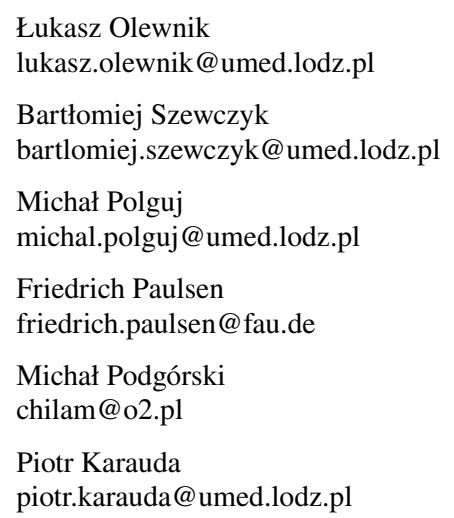

1 Department of Anatomical Dissection and Donation, Medical University of Lodz, Lodz, Poland

2 Department of Normal and Clinical Anatomy, Chair of Anatomy and Histology, Medical University of Lodz, Lodz, Poland

3 Polish Mother's Memorial Hospital Research Institute, Lodz, Poland

4 Institute of Functional and Clinical Anatomy, Erlangen, Germany

5 Department of Topographic Anatomy and Operative Surgery, Sechenov University, Moscow, Russia

6 Laboratory of Anatomy, Faculty of Medicine, Rouen University, Mont-Saint-Aignan, France 


\section{Introduction}

The coracoid process serves as an important anchor for several tendinous and ligamentous structures. These include, medially to laterally, the tendons of the pectoralis minor, coracobrachialis (CRM), and the short head of the biceps brachii muscles (shBB), and, laterally to medially, the coracohumeral, coracoacromial, coracoclavicular, and superior transverse scapular ligaments. The CRM and shBB share a common origin on the apex of the coracoid process of the scapula. The CRM inserts into the medial surface of the humerus, between the attachments of the triceps brachii and brachialis muscles by means of a short, flat tendon [1]. The CRM serves to flex and adduct the arm at the glenohumeral joint, and to resist deviation of the arm from the frontal plane during abduction [1].

The brachial plexus and the major axillary vessels run medially and inferiorly to the coracoid process; they begin to divide into branches from the medial, lateral, and posterior cords at the level of the coracoid process, anterior to the inferior glenoid [1]. The lateral cord of the brachial plexus gives rise to the musculocutaneous nerve $(\mathrm{MCN})$, which contains fibers from the $\mathrm{C} 5-\mathrm{C} 7$ ventral rami. The $\mathrm{MCN}$ passes through the CRM and descends between the biceps brachii and brachialis muscles, innervating both of them [1].

A number of authors have described variations in the CRM and MCN [2-8]. Most of these classifications are based on whether the MCN pierces CRM or not; however, that of Loukas et al. [7] includes the relationship between the MCN, the median nerve (MN) and the CRM. In contrast, Hayashi et al. [9] examined the relationship between the communicating branch and the transposed innervation of the brachial flexors to the median nerve. Only El-Naggar et al. [2] and Ilayperuma et al. [10], examined the morphology of the muscle. The remaining studies mainly concern reports of isolated cases, such as examples of accessory slips of the muscle inserting to the medial epicondyle and medial supracondylar ridge of the humerus, or additional heads or bellies [6, 11-14].

A good understanding of the anatomical relationship between the coracoid process and the CRM, shBB and pectoralis minor, as well as other glenohumeral joint supporting structures, is needed to correctly interpret shoulder magnetic resonance (MR) imaging of this area, and when planning proper surgical procedures in this area.

The aim of the present study was to characterize possible variations in the morphology of the proximal and distal attachments of the CRM and to draw conclusions from this with regard to an accurate classification of the area that can be useful for planning surgical procedures in the region. It also should assess the relationship between CRM type and MCN course.

\section{Materials and methods}

One hundred and one upper limbs (52 left, and 49 right) fixed in $10 \%$ formalin solution were examined. The mean age "at death" of the cadavers was 77.1 years (48-95), and the group comprised equal numbers of female and male adults (Central European population). The cadavers were the property of the Department of Anatomical Dissection and Donation, Medical University of Lodz, Poland, following donation to the university anatomy program. Any upper limbs with evidence of surgical intervention in the dissected area were excluded. All dissection of the shoulder and arm area were performed in accordance with an pre-established protocol [15-19].

Dissection began with the removal of the skin and superficial fascia from the area of the shoulder and medial side of the arm. The next step included lateral, medial and posterior cords of the brachial plexus visualization, as well as accurate visualization of both biceps brachii, CRM and brachialis muscle. Following this, all structures were thoroughly cleaned.

Upon dissection, the following morphological features of the CRM were assessed:

- The type of origin of the CRM

- The type of insertion of the CRM

- The relationship between CRM and MCN

- Morphometric measurements of the CRM and MCN.

When dissecting the CRM:

- Special attention was paid when cleaning the shBB as it has numerous connections to the CRM, and provides an origin for the CRM.

- when assessing the course of the MCN, the deep head of the CRM was often invisible at first sight: the area was thoroughly cleaned.

An electronic digital caliper was used for all measurements (Mitutoyo Corporation, Kawasaki-shi, Kanagawa, Japan), and each measurement was performed twice with an accuracy of up to $0.1 \mathrm{~mm}$. The Bioethics Committee of the Medical University of Lodz (resolution RNN/1337/20/ $\mathrm{KE}$ ) approved the study protocol. The cadavers belong to the Department of Anatomical Dissection and Donation of the Medical University of Lodz, Poland.

\section{Statistical analysis}

Statistica 13 software (StatSoft Polska, Cracow, Poland) was used for the statistical analysis. The following tests were applied: 
- The Chi-square test to compare nominal data-differences of muscle, insertion and innervation types between each other and between body sides and sexes.

- The Shapiro-Wilk test to assess normality of the morphological measurments distribution. As the data was not normally distributed nonparametric tests were used.

- The Mann-Whitney test to compare morphological measurements between body sides, sex, and types of insertion and innervation.

- The Kruskal-Wallis ANOVA by ranks with dedicated post hoc test to compare measurements between muscle types.

A p-value lower than 0.05 was considered significant, with Bonferroni's correction for multiple testing. The results are presented as mean and standard deviation unless otherwise stated.

\section{Results}

The CRM was present in all 101 dissected limbs. The observed anatomical variations were grouped according to the following categories:

- Type of muscle origin

- Type I (49.5\%)—single belly originating from the coracoid process, medially and posteriorly to the tendon of the shBB (50 cases: 30 female and 20 male; 22 right and 28 left)—Figs. 1, 4a

- Type II (42.6\%)—double muscle belly originating from-Fig. 2.

- Type IIa-one head originating from the coracoid process posteriorly to the tendon of the biceps brachii and a second head originating from the shBB (22 cases: 13 females and 9 males; 11 right and 11 left)-Figs. 2a, 4b.

- Type IIb-both heads originating from the coracoid process; however, the superficial head fuses with the insertion of the shBB, while the deep head is directly originating ( 21 cases: 17 female and four male; 12 right and 9 left)—Figs. 2b, 4c.

- Type III (7.9\%) - three heads, two originating from the coracoid process (superficial and deep), whereas the third originates from the shBB (eight cases: eight males; four right and four left)Figs. 3, 4d.

- Type of insertion

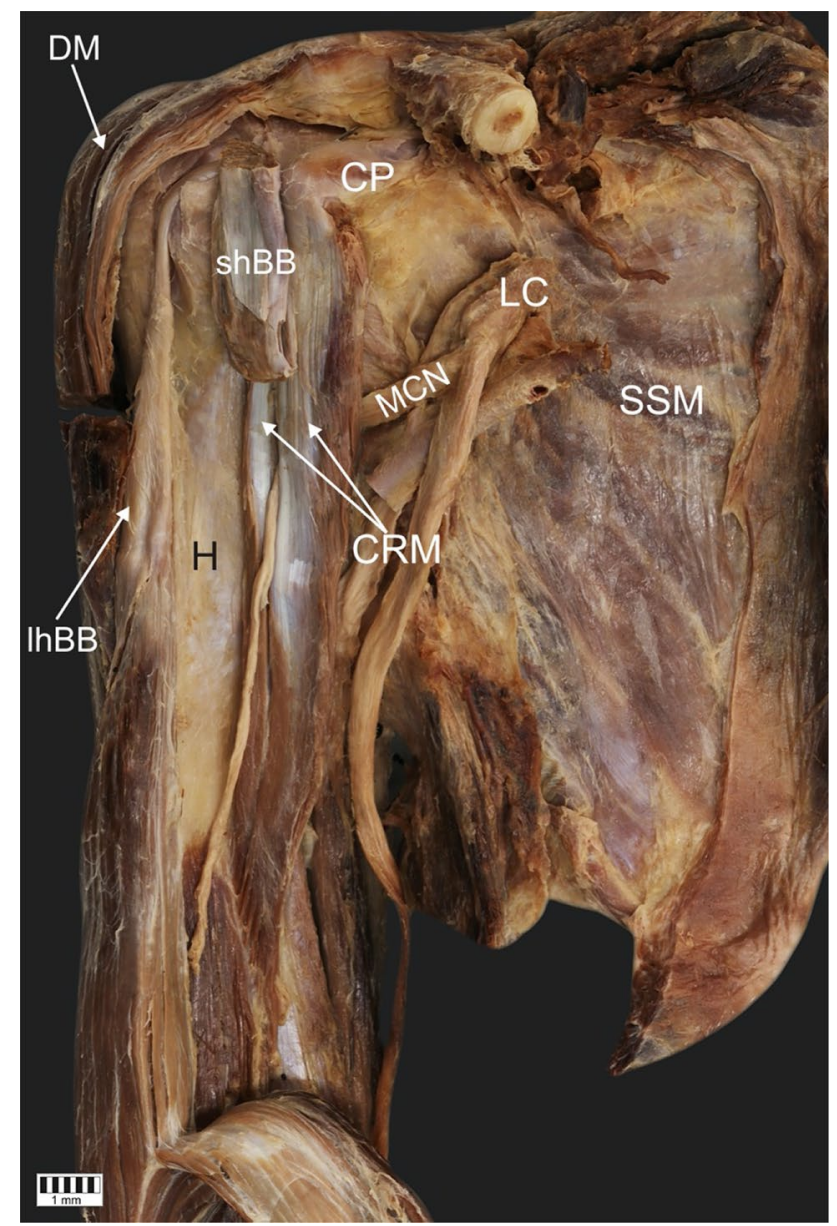

Fig. 1 Type I of origin of the coracobrachialis muscle. Right arm. $D M$ deltoid muscle, $C P$ coracoid process of the scapula, $s h B B$ short head of the biceps brachii, $\operatorname{lhBB}$ long head of the biceps brachii, $L C$ lateral cord of the brachial plexus, $H$ humerus, SSM subscapularis muscle, $M C N$ musculocutaneous nerve, $C R M$ coracobrachialis muscle

- Type 1-single, classical insertion on the distal 1/3 of the humerus (61 cases: 32 female and 29 male; 29 right and 32 left)—Fig. 5.

- Type 2-double insertion: one on the distal $1 / 3$ of the humerus and another fusing with the medial head of the triceps brachii ( 40 cases: 28 females and 12 males; 20 right and 20 left)—Fig. 6.

- Relation to the MCN

- Type I-it pierces the muscle belly (50 cases: 30 females and 20 males; 22 right and 28 left).

- Type II-it passes between the heads of the coracobrachialis (51 cases: 30 females and 21 males; 27 right and 24 left). 


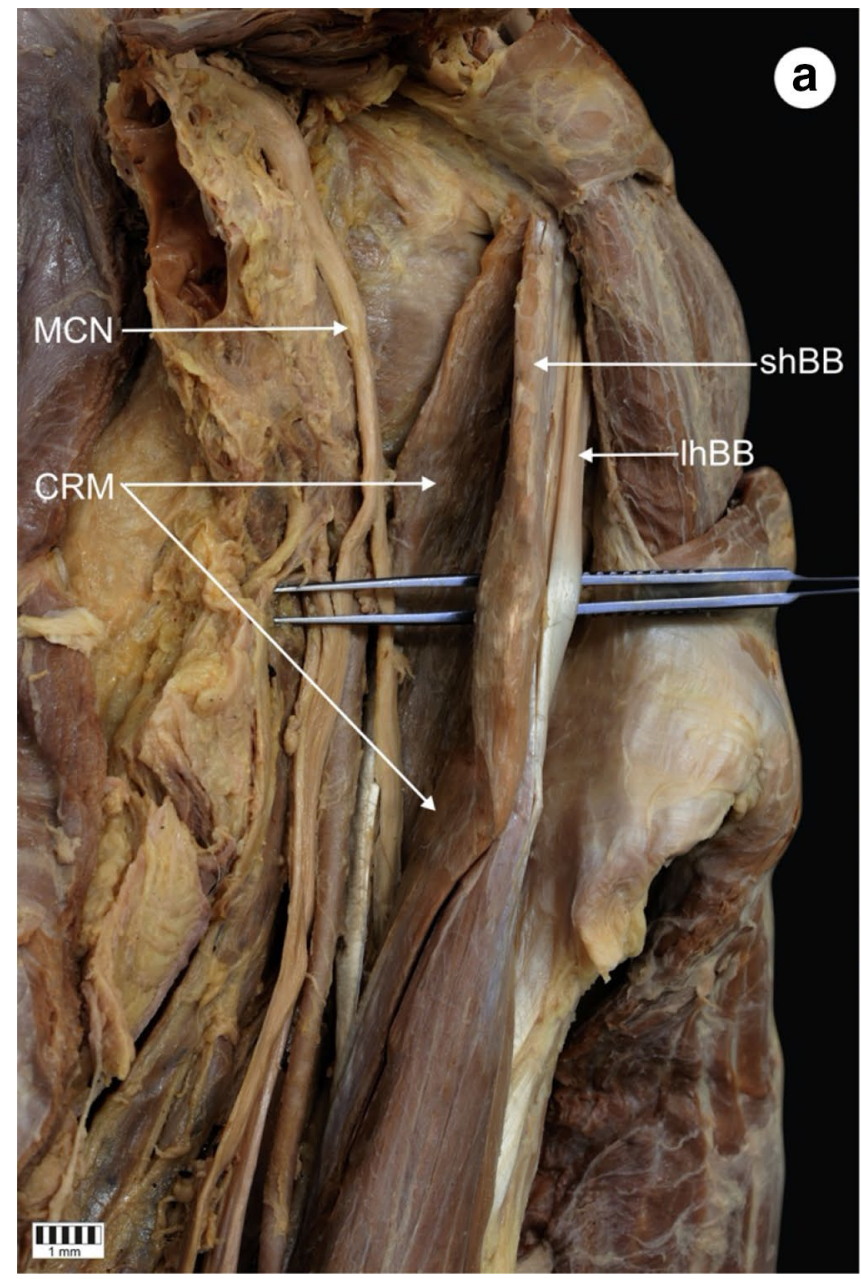

Fig. 2 Type II of origin of the coracobrachialis muscle. Left arm. a Type IIa of the coracobrachialis muscle. $M C N$ musculocutaneous nerve, $C R M$ coracobrachialis muscle, $s h B B$ short head of the biceps brachii, $l h B B$ long head of the biceps brachii, $C P$ coracoid process.

A significant difference in origin type was observed between sexes $(p=0.0003)$ but not between body sides $(p=0.7861)$. However, no such differences were observed for insertion types $(p=0.1215$ for sexes and $p=0.9495$ for body sides) nor for the relationship with the MCN ( $p=0.9344$ for sexes and $p=0.4840$ for body sides). Interestingly, only in one case of a double-headed CRM was the MCN found to pierce the muscle instead of passing between the two heads.

Morphometric parameters are presented according to sex and body side in Table 1, and according to insertion type and relationship to MCN in Table 2. In addition, they are presented with regard to origin type in Table 3.

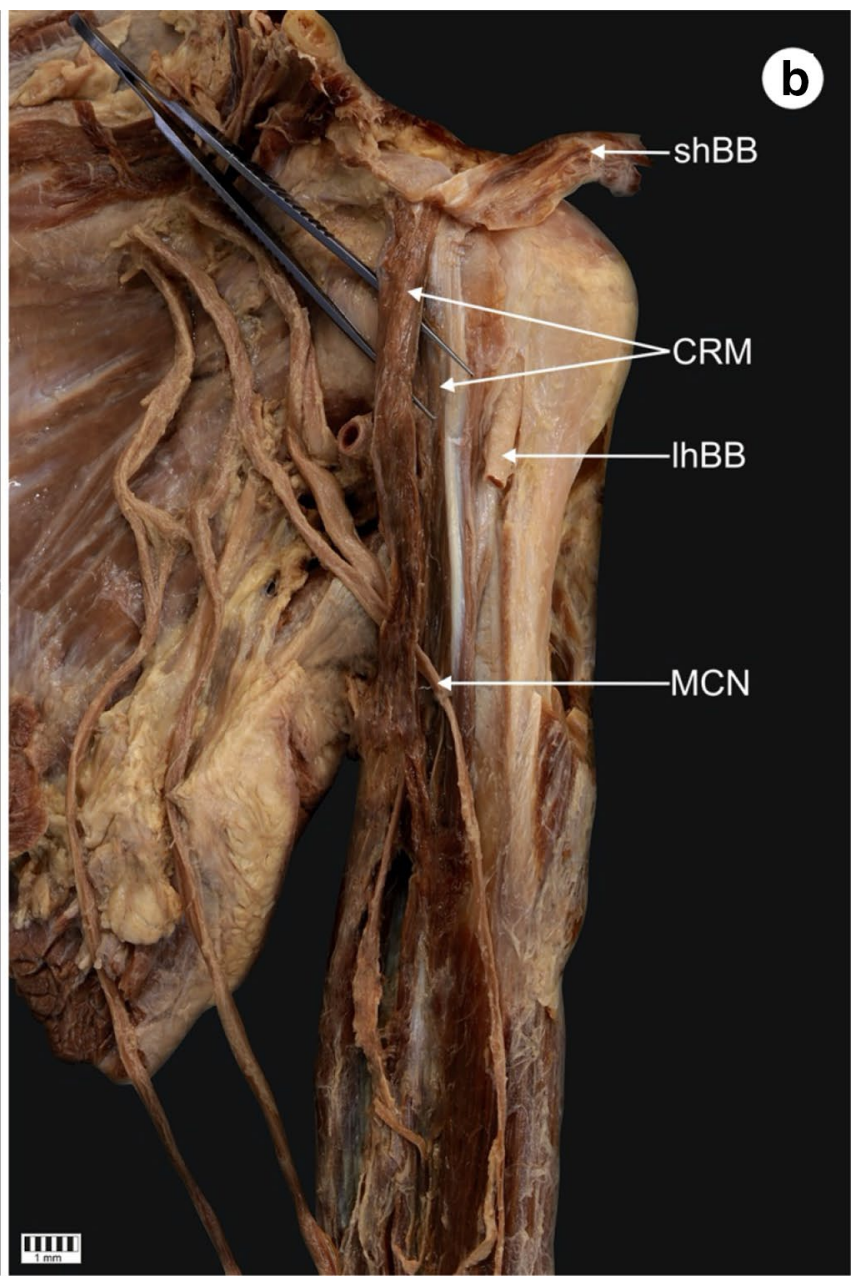

b Type IIb of origin of the coracobrachialis muscle. CRM coracobrachialis muscle, $\operatorname{sh} B B$ short head of the biceps brachii, $\operatorname{lh} B B$ long head of the biceps brachii, $M C N$ musculocutaneous nerve, $C P$ coracoid process

\section{Discussion}

The key value of the present work is that it presents a new systematic classification of CRM origin and insertion based on anatomical dissection. In addition, it assesses the relationship between individual types of CRM and MCN.

To understand the occurrence of CRM variations, it is necessary to review their embryological development. Embryologically, the biceps brachii, CRM, and brachialis muscle are believed to arise from a common premuscular mass. The origins of the two heads of the biceps brachii become separated as the scapula develops. The 


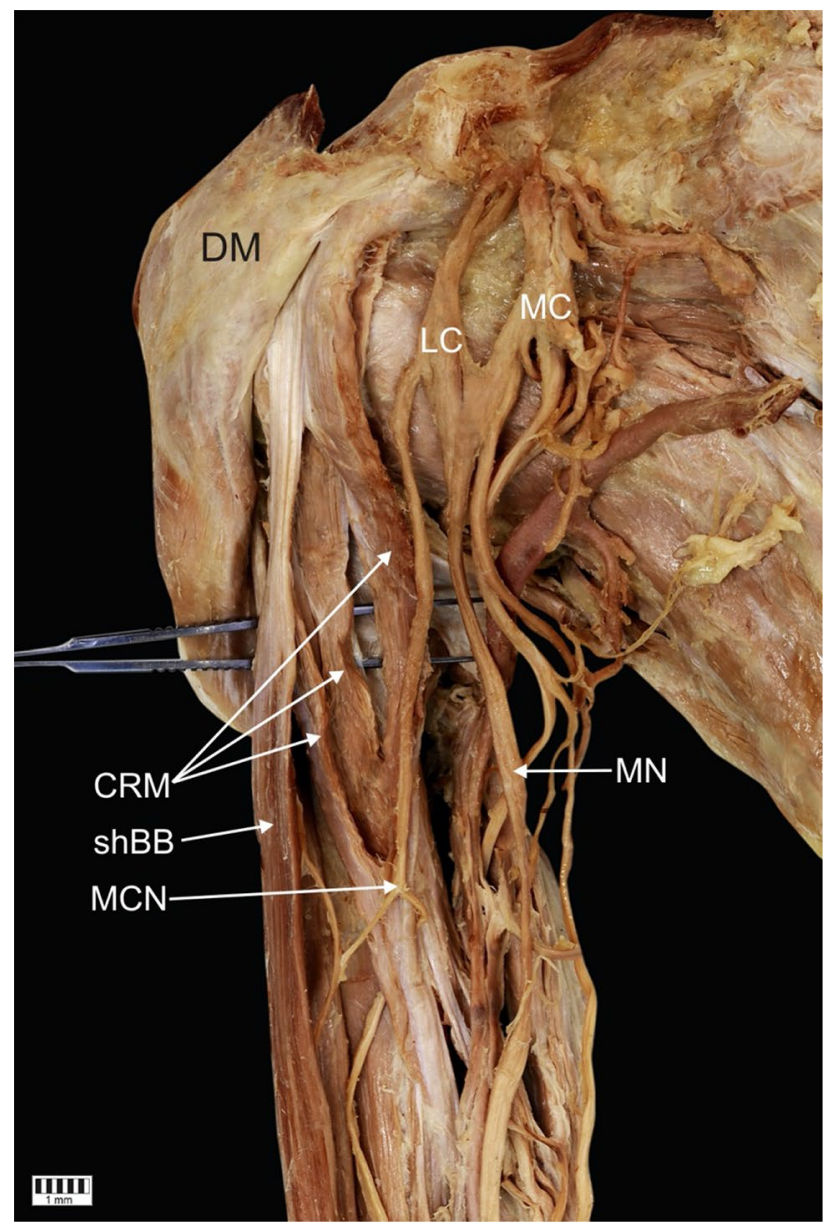

Fig. 3 Type III of origin of the coracobrachialis muscle. Right arm. $D M$ deltoid muscle, $L C$ lateral cord of the brachial plexus, $M C$ medial cord of the brachial plexus, $C R M$ coracobrachialis muscle, $s h B B$ short head of the biceps brachii, $M C N$ musculocutaneous nerve, $M N$ median nerve, $C P$ coracoid process three muscles can be recognized in embryos 14-16 mm in length, and the tendon of the long head in embryos $14 \mathrm{~mm}$ in length. The distal end of the common muscle mass differentiates later than the proximal end [20, 21]. The presence of the CBL could be explained as a result of the premature termination of this regression process.

Little information exists about the morphological variability of the proximal attachment of the CRM [2, 10]. Most likely, the first morphological variability of CRM was described by Wood [22], who describes coracocapsularis originating from the coracoid process and inserting into the shoulder capsule [22]. El-Naggar reports that the CRM consists of two heads; a superficial (anterior) head and a deep (posterior) head [2]. The superficial head originates from the medial border of the tendon of the shBB, while the deep head originates from the coracoid process of the scapula and the adjoining part of the lateral border of the tendon of the shBB [2]. The deep layer of the coracobrachialis can originate from the insertion of the pectoralis major [23]. Interestingly, one case has been reported of a three-headed CRM, characterized by a single superficial head and a deep head split into two [2]. In contrast, Ilayperuma et al. [10] do not report any such morphological variations in the CRM proximal attachment: they describe three possible proximal attachments for a single belly relative to the tendon of the biceps brachii[10], these being lateral to the tendon, medial to the tendon and deep to the origin of the tendon of the biceps brachii. Cases of accessory CRM have also been observed, which typically originates from the posterior margin of the coracoid process and inserts into the tendinous part of latissimus dorsi (the coracobrachialis minor or secundus) [22, 24]. Olewnik et al. [25] found a really rare case of CRM, which was characterized by four heads. The first two heads of the CRM demonstrate a proximal attachment at "the accessory apex" of the coracoid process, the third

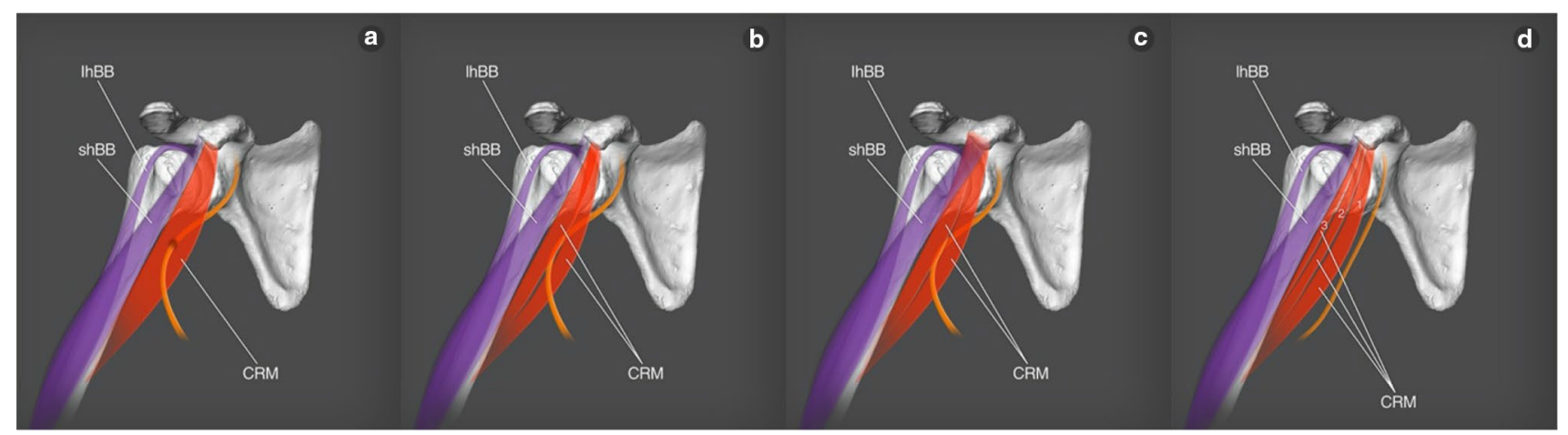

Fig. 4 Scheme of types origin of coracobrachialis muscle. a scheme of Type I origin of coracobrachialis muscle $\operatorname{lh} B B$ long head of the biceps brachii $s h B B$ short head of the biceps brachii $C R M$ coracobrachialis muscle $\mathbf{b}$ scheme of Type II a origin of coracobrachialis muscle $l h B B$ long head of the biceps brachii $\operatorname{sh} B B$ short head of the biceps brachii $C R M$ coracobrachialis muscle $\mathbf{c}$ scheme of Type II b origin of coracobrachialis muscle $\operatorname{lh} B B$ long head of the biceps brachii $\operatorname{sh} B B$ short head of the biceps brachii $C R M$ coracobrachialis muscle $\mathbf{d}$ scheme of Type III origin of coracobrachialis muscle $\operatorname{lh} B B$ long head of the biceps brachii $\operatorname{sh} B B$ short head of the biceps brachii $C R M$ coracobrachialis muscle 


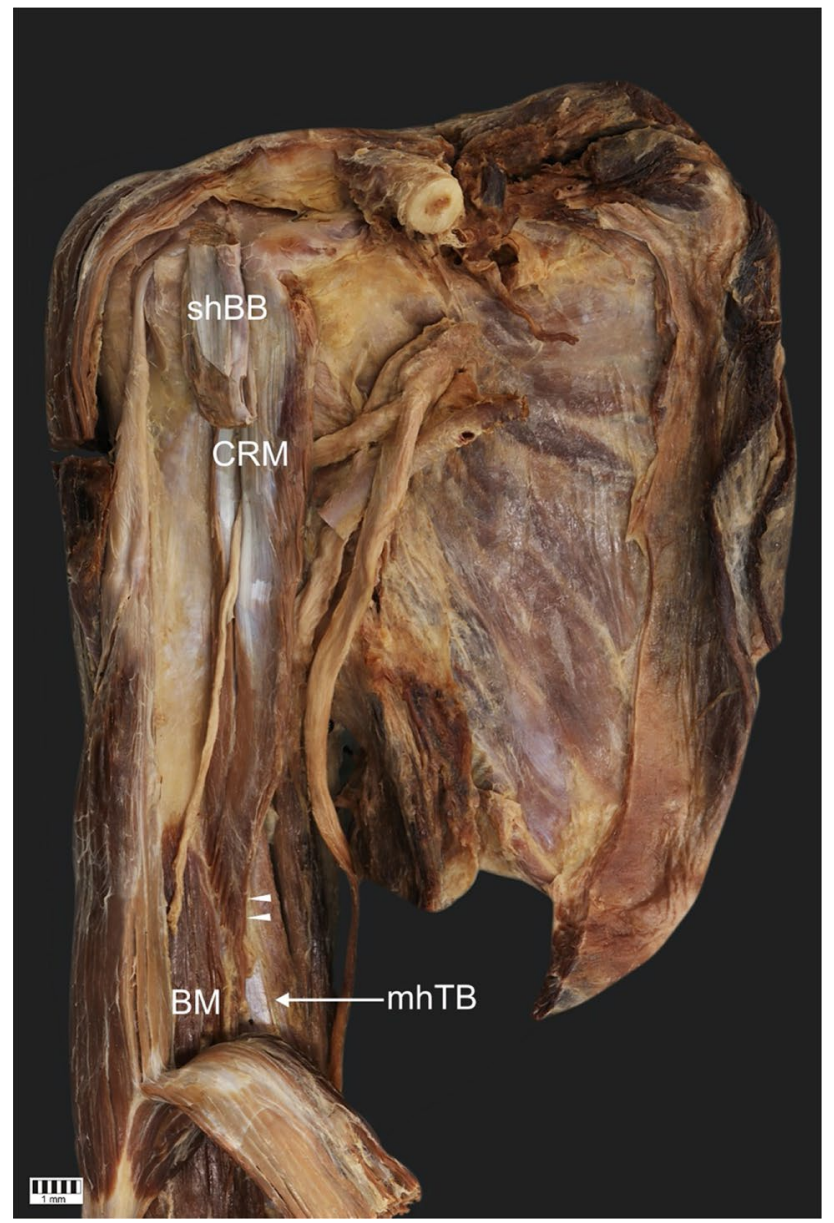

Fig. 5 Type 1 of insertion of the coracobrachialis muscle. Right arm. $s h B B$ short head of the biceps brachii, $C R M$ coracobrachialis muscle, $B M$ brachialis muscle, $m h T B$ medial head of the triceps brachii, $C P$ coracoid process, $C$ clavicle, white arrowheads show the insertion of the coracobrachialis muscle

head, together with the head of the shBB, was attached to the apex of the coracoid process and was characterized by a fusion with the shBB; the fourth head was located under the head of the shBB and displayed an attachment at the inferior surface of the coracoid process [25]. The fourth head in the distal part demonstrated a fusion to the brachialis muscle, with the distal attachment being at the middle of the medial surface and the border of the body of the humerus, together with the other three heads [25].

In very rare cases, a coracobrachialis longus muscle may be observed $[12,13,16]$, with the CRM being absent $[22$, 26].

However, a new classification is needed for clinical, anatomical and didactic reasons. The present study proposes a new threefold CRM classification (Types I-III), with Type II being further divided into two subtypes (A-B). The proposed classification is based on the number of bellies: Type I, present in $49.5 \%$ of cases, is characterized by a single

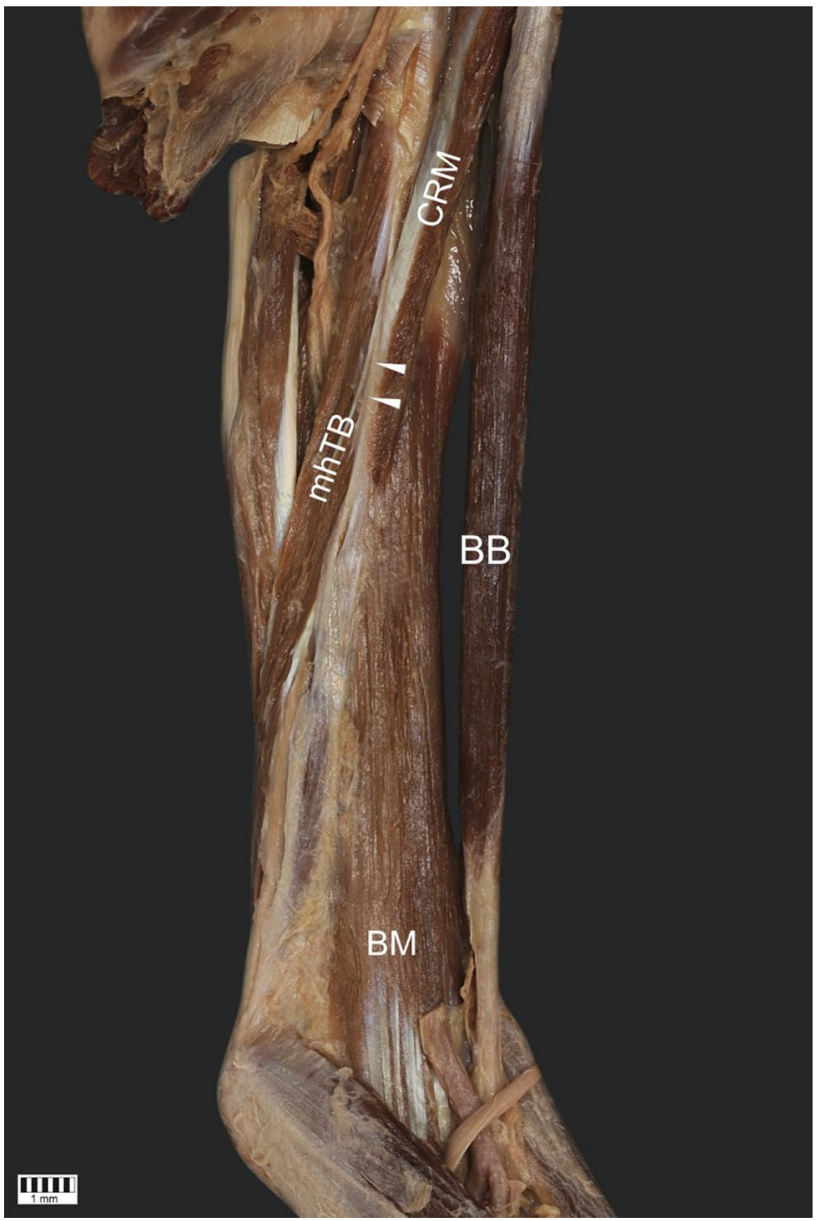

Fig. 6 Type 2 of insertion of the coracobrachialis muscle. $m h T B$ medial head of the triceps brachii, $C R M$ coracobrachialis muscle, $B B$ biceps brachii, $B M$ brachialis muscle, $M E$ medial epicondyle of the humerus, white arrowheads show fusion between coracobrachialis and medial head of the triceps brachii

belly with the proximal attachment located on the coracoid process, medially and posteriorly to the tendon of the shBB. Type II (42.6\%) is characterized by the occurrence of two bellies. This type was divided into two subtypes: A and B. In Type IIA, the first head originates from the coracoid process posterior to the tendon of the shBB and the second head originates from the shBB. In Type IIB, both heads originate from the coracoid process; however, the superficial head fuses with the insertion of the shBB, while the deep head is directly inserted. This Type IIB corresponds to the type described by El-Nagger [2]. Type III is characterized by a three-headed CRM (7.9\%): two heads (superficial and deep) originate from the coracoid process, whereas the third arises from the shBB. It is worth noting that this type of CRM has not been previously described in other studies on the variability of this muscle $[2,10]$.

It may seem that this small, inconspicuous muscle may have a much greater clinical significance than previously 
Table 1 Morphometric parameters according to sex and bodyside

\begin{tabular}{|c|c|c|c|c|c|c|c|}
\hline \multirow[t]{2}{*}{ Parameter } & \multirow[t]{2}{*}{ Head } & \multicolumn{2}{|l|}{ Sex } & \multirow[t]{2}{*}{$P$ value } & \multicolumn{2}{|l|}{ Body side } & \multirow[t]{2}{*}{$P$ value } \\
\hline & & Female & Male & & Right & Left & \\
\hline \multirow[t]{3}{*}{ Muscle belly length } & 1 & $103.92(18.32)$ & $113.10(19.27)$ & 0.0211 & $107.84(19.34)$ & $107.46(19.17)$ & 0.7832 \\
\hline & 2 & 87.85 (25.69) & $81.94(33.31)$ & 0.4554 & $86.61(27.41)$ & $84.08(31.04)$ & 0.6989 \\
\hline & 3 & & $100.83(12.65)$ & - & $100.83(12.61)$ & $100.83(14.64)$ & 0.8852 \\
\hline \multirow[t]{3}{*}{ Origin width } & 1 & $7.15(2.24)$ & $7.80(1.72)$ & 0.0424 & $7.45(2.17)$ & $7.38(1.98)$ & 0.6885 \\
\hline & 2 & $9.81(3.87)$ & $11.15(5.43)$ & 0.7312 & $10.27(4.74)$ & $10.53(4.54)$ & 0.9718 \\
\hline & 3 & & $14.77(3.69)$ & - & $14.33(3.92)$ & $15.21(3.98)$ & 0.4705 \\
\hline \multirow[t]{3}{*}{ Origin thickness } & 1 & $2.46(0.70)$ & $2.57(0.66)$ & 0.3330 & $2.50(0.74)$ & $2.51(0.64)$ & 0.9702 \\
\hline & 2 & $1.76(0.71)$ & $1.87(0.53)$ & 0.2135 & $1.87(0.68)$ & $1.72(0.59)$ & 0.5169 \\
\hline & 3 & & $1.77(1.41)$ & - & $1.63(1.20)$ & $1.91(1.77)$ & 0.8852 \\
\hline Coracobrachialis tendon length & - & $40.14(13.08)$ & $41.02(14.70)$ & 0.8900 & $42.02(13.88)$ & $39.06(13.51)$ & 0.2565 \\
\hline Muscle width in junction & - & $5.00(1.51)$ & $5.70(1.54)$ & 0.0264 & $5.39(1.50)$ & $5.19(1.61)$ & 0.5319 \\
\hline Muscle thickness in junction & - & $2.23(0.58)$ & $2.36(1.04)$ & 0.8600 & $2.32(0.83)$ & $2.26(0.77)$ & 0.4148 \\
\hline $\begin{array}{l}\text { Distance between musculocutaneous } \\
\text { nerve branching and piercing/passing } \\
\text { through the muscle }\end{array}$ & - & $69.36(22.68)$ & $74.23(24.90)$ & 0.3523 & $71.74(23.74)$ & $70.96(23.71)$ & 0.9107 \\
\hline \multirow[t]{2}{*}{ Musculocutaneous nerve diameter } & Before muscle & $3.14(1.08)$ & $3.22(0.86)$ & 0.3742 & $3.25(1.09)$ & $3.10(0.90)$ & 0.5055 \\
\hline & After muscle & $2.81(1.03)$ & $2.92(0.82)$ & 0.2822 & $2.96(1.10)$ & $2.75(0.78)$ & 0.4246 \\
\hline
\end{tabular}

$p$-values lower than 0.0033 are significant, according to Bonferroni’s correction

Table 2 Morphometric parameters according to type of insertion and relationship with MCN

\begin{tabular}{|c|c|c|c|c|c|c|c|}
\hline \multirow[t]{2}{*}{ Parameter } & \multirow[t]{2}{*}{ Head } & \multicolumn{2}{|c|}{ Type of insertion } & \multirow[t]{2}{*}{$P$ value } & \multicolumn{2}{|c|}{$\begin{array}{l}\text { Relation to musculocutaneous } \\
\text { nerve }\end{array}$} & \multirow[t]{2}{*}{$P$ value } \\
\hline & & Type 1 & Type 2 & & Type 1 & Type 2 & \\
\hline \multirow[t]{3}{*}{ Muscle belly length } & 1 & $104.16(19.29)$ & $112.96(17.91)$ & 0.0327 & 104.24 (18.69) & $110.98(19.21)$ & 0.0275 \\
\hline & 2 & $70.43(21.91)$ & $101.01(27.27)$ & 0.0004 & $54.21(0.00)$ & $86.04(28.85)$ & 1.0000 \\
\hline & 3 & $95.35(8.94)$ & $117.26(0.89)$ & 0.0668 & & $100.83(12.65)$ & - \\
\hline \multirow[t]{3}{*}{ Origin width } & 1 & 7.021 .53() & $8.01(2.58)$ & 0.0268 & $7.74(2.33)$ & $7.09(1.72)$ & 0.2987 \\
\hline & 2 & $8.34(2.93)$ & $12.09(5.07)$ & 0.0017 & $11.21(0.00)$ & $10.37(4.65)$ & - \\
\hline & 3 & $15.53(4.02)$ & $12.48(0.71)$ & 0.2433 & & 14.77 (3.69) & - \\
\hline \multirow[t]{3}{*}{ Origin thickness } & 1 & $2.32(0.60)$ & $2.77(0.73)$ & 0.0020 & $2.48(0.61)$ & $2.53(0.76)$ & 0.4150 \\
\hline & 2 & $1.91(0.70)$ & $1.71(0.58)$ & 0.1539 & $3.32(0.00)$ & $1.77(0.60)$ & - \\
\hline & 3 & $1.04(0.28)$ & $3.96(0.82)$ & 0.0668 & & $1.77(1.41)$ & - \\
\hline Coracobrachialis tendon length & - & $43.01(14.51)$ & $36.67(11.52)$ & 0.0430 & $39.57(14.84)$ & $41.40(12.56)$ & 0.2801 \\
\hline Muscle width in junction & - & $5.32(1.50)$ & $5.23(1.65)$ & 0.7337 & $5.26(1.68)$ & $5.31(1.44)$ & 0.8016 \\
\hline Muscle thickness in junction & - & $2.27(0.86)$ & $2.32(0.70)$ & 0.5366 & $2.34(0.84)$ & $2.23(0.75)$ & 0.6152 \\
\hline $\begin{array}{l}\text { Distance between musculocutaneous } \\
\text { nerve branching and piercing/passing } \\
\text { through the muscle }\end{array}$ & - & $77.75(24.20)$ & $61.56(19.12)$ & 0.0012 & $71.00(26.89)$ & $71.67(20.15)$ & 0.6010 \\
\hline \multirow[t]{2}{*}{ Musculocutaneous nerve diameter } & Before muscle & $3.01(0.79)$ & $3.42(1.21)$ & 0.0319 & $3.09(0.89)$ & $3.25(1.09)$ & 0.4016 \\
\hline & After muscle & $2.78(0.77)$ & $2.96(1.18)$ & 0.4246 & $2.77(0.88)$ & $2.93(1.02)$ & 0.3131 \\
\hline
\end{tabular}

$p$-values lower than 0.0033 are significant, according to Bonferroni's correction

thought. It has been speculated that CRM is functionally not important; however, some studies suggest that it may be one of the most effective flexors of the shoulder joint and that it resists anterior dislocation [27]. The shoulder is the most regularly dislocated joint in the body, with dislocation occurring anteriorly, posteriorly, inferiorly, or anterior-superiorly. Of these, anterior locations are the most common, occurring in $95-97 \%$ of cases [28-30]. Patients with prior shoulder 
Table 3 Morphometric parameters according to origin type and insertion type

\begin{tabular}{|c|c|c|c|c|c|c|}
\hline \multirow[t]{3}{*}{ Parameter } & \multirow[t]{3}{*}{ Head } & \multicolumn{4}{|l|}{ Type of origin } & \multirow[t]{3}{*}{$P$ value } \\
\hline & & \multirow[t]{2}{*}{ Type 1} & \multicolumn{2}{|l|}{ Type 2} & \multirow[t]{2}{*}{ Type 3} & \\
\hline & & & $\mathrm{a}$ & $\mathrm{b}$ & & \\
\hline \multirow[t]{3}{*}{ Muscle belly length } & 1 & $103.76(19.16)$ & $109.74(23.52)$ & $108.96(14.39)$ & $122.70(6.28)$ & 0.0281 \\
\hline & 2 & - & $75.63(31.14)$ & 102.67 (16.57) & $67.05(26.32)$ & 0.0020 \\
\hline & 3 & - & - & - & $100.83(12.65)$ & - \\
\hline \multirow[t]{3}{*}{ Origin width } & 1 & $7.80(2.33)$ & $6.97(1.80)$ & $6.70(1.52)$ & $8.09(1.66)$ & 0.1908 \\
\hline & 2 & - & $11.24(6.25)$ & $11.02(3.55)$ & $7.14(0.80)$ & 0.0330 \\
\hline & 3 & - & - & - & 14.77 (3.69) & - \\
\hline \multirow[t]{3}{*}{ Origin thickness } & 1 & $2.48(0.61)$ & $2.41(0.76)$ & $2.75(0.78)$ & $2.24(0.61)$ & 0.3363 \\
\hline & 2 & - & $1.92(0.83)$ & $1.71(0.54)$ & $1.84(0.48)$ & 0.6988 \\
\hline & 3 & - & - & - & $1.77(1.41)$ & - \\
\hline Coracobrachialis tendon length & - & $39.50(14.74)$ & $46.00(11.67)$ & $40.44(12.29)$ & $31.72(11.40)$ & 0.0308 \\
\hline Muscle width in junction & - & $5.31(1.69)$ & $5.44(1.38)$ & $5.32(1.54)$ & $4.64(1.28)$ & 0.6000 \\
\hline Muscle thickness in junction & - & $2.33(0.84)$ & $2.03(0.57)$ & $2.52(0.88)$ & $2.13(0.75)$ & 0.4044 \\
\hline $\begin{array}{l}\text { Distance between musculocutaneous nerve } \\
\text { branch and passage through the muscle }\end{array}$ & - & $71.60(27.10)$ & $72.00(23.53)$ & $66.73(17.60)$ & $79.93(11.21)$ & 0.4841 \\
\hline \multirow[t]{2}{*}{ Musculocutaneous nerve diameter } & Before muscle & $3.09(0.89)$ & $2.92(0.63)$ & $3.59(1.42)$ & $3.35(0.92)$ & 0.1981 \\
\hline & After muscle & $2.76(0.88)$ & $2.74(0.62)$ & $3.17(1.41)$ & $2.90(0.48)$ & 0.5463 \\
\hline
\end{tabular}

$p$-values lower than 0.003 are significant according to Bonferroni's correction

dislocation are more prone to redislocation, which typically occurs due to the tissue not healing properly or losing tension. Proximal rupture of the CRM can also hasten anterior dislocation of the shoulder [31]. It remains unclear, however, whether a CRM with two bellies is more likely to predispose the bearer to anterior dislocation than one with three bellies. Furthermore, the origin of the CRM from the shBB strongly indicates that CRM acts as a muscle enhancer for the shBB (Type IIa, III). A new CRM classification is needed. This would be a good step towards potentially extending the classification to "rare cases" in the upper and lower limbs, as was the case with earlier classifications [32-39].

The distal attachment of the CRM was usually observed on the medial border of the diaphysis of the humerus between the attachments of the medial head of the triceps brachii and the brachialis muscle $[2,10]$. This type of insertion (Type 1) was observed in $60.4 \%$ of all cases in the present study. Type 2 characterized by a double insertion on the distal $1 / 3$ of the humerus and fusion with the medial head of triceps brachii was observed in $39.6 \%$ of cases. Interestingly, it was found that the simultaneous proximal attachment to the shBB co-occurred with a simultaneous distal attachment to the medial head of the triceps brachii. However, the function of a muscle that demonstrates attachments to antagonistic muscles remains unclear.

Can a CRM with two or three bellies be used as a source of material in plastic surgery? Type IIa demonstrated the longest tendon (46 mm mean length) and could possibly be used to reconstruct other tendons and ligaments.
MCN neuropathy is not as common as MN, ulnar or radial neuropathy. It has a similar course to CRM neuropathy and can pierce or pass deep to the CRM [2, 7, 10, 40, 41]. The CRM is thought to be the most common site of MCN entrapment, and additional heads can place pressure on the MCN [4-6, 11, 12, 14]. MCN entrapment within the CRM muscle results in weakness and atrophy of the biceps brachii and brachialis muscles and a loss of sensation in the lateral forearm. Active young individuals that frequently engage in shoulder and elbow flexion with the forearm in a pronated position are most susceptible [42]. It also often occurs following chronic overuse of the CRM and consequent hypertrophy. No loss of CRM function will be observed as the nerve compressed within the CRM has already given off its motor branch to the CRM.

The present study outlines two types of innervation in relation to the CRM. In Type 1 the MCN pierces the CRM and is strongly associated with Type I muscle morphology (100\% of cases). In contrast, in Types II and III, the MCN passes between the CRM heads. Interestingly, only in one case of a double CRM head did the MCN pierce the muscle instead of passing between the heads. The frequency of the atypical course and relationship of the MCN to the CRM has been exhaustively described in the literature [2, 3, 7, $10,40,41,43]$. The MCN innervates the CRM in 0 to $22 \%$ of cases [2, 4, 7, 10, 40, 41]. Interestingly, while previous studies found the CRM to not be pierced by the MCN in less than $7 \%$ of cases $[8,26,44]$, the present study found it to be the case in $51 \%$. It is possible that previous studies do not 
reveal the potential types of CRM or omit the deep layer, or that these differences result only from recently described population differences.

The present study has some limitations. One is the heterogeneous nature of the classification, which depends on several morphological details such as type of insertion or origin. In addition, it is only an anatomical study, and so a spectrum of variation could be presented; further studies should examine the potential value of ultrasound or MRI for this purpose. Nonetheless, this study helps raise awareness of what to look for, and where to find it, and offers a uniform classification and terminology, which can be used as a foundation for communication with surgeons.

Nevertheless, the proposed classification has four key assets. First, it recognizes the different possibilities of proximal attachment of the CRM. It also highlights the variety of distal attachments. It demonstrates the variable course of MCN relative to given types of CRM morphology (Type I-III). It also proposes a systematic classification of CRM morphological variability.

In addition, a thorough understanding of the CRM is needed for effective treatment and rehabilitation of anterior dislocation shoulder or MCN neuropathy, and hence our findings have offered new data for anatomy, physiotherapy, and orthopedic surgery. They can also be used in the future for the reconstruction of other tendons or ligaments, as well as support plastic surgery.

\section{Conclusion}

The CRM is characterized by high morphological variability. The new classification proposes three types of proximal attachment (I-III) and two types of distal attachment. In addition, two types of MCN are distinguished. While Type I CRM is always pierced by the MCN, Types II and III are pierced in only one case. This inconspicuous muscle can be of great clinical importance, and our proposed classification may be of great value to surgeons operating in this area.

\footnotetext{
Author contributions BS (D.P.T.) - assistant-project development, data collection and management, data analysis and manuscript writing. MP (MD., PhD)—Professor-data analysis, manuscript editing. FP (MD., PhD) — professor-data analysis, manuscript editing. MP (MD., PhD) — associate professor-statistical analysis, data analysis and manuscript editing. FD (MD., PhD) - professor-data analysis, manuscript editing. PK-Assistant-data analysis, manuscript editing. $Ł O$ (D.P.T., PhD) —Associate Professor-data collection and management, data analysis and manuscript editing. All authors have read and approved the manuscript.
}

Funding The authors have no financial or personal relationship with any third party whose interests could be positively or negatively influenced by the article's content. This research did not receive any specific grant from funding agencies in the public, commercial, or not-for-profit sectors.

Availability of data and materials Please contact authors for data requests (Łukasz Olewnik PhD—email address: lukasz.olewnik@ umed.lodz.pl).

\section{Compliacne with ethical standards}

Conflict of interest The authors declare that they have no competing interests. Financial Disclosure: Friedrich Paulsen receives royalties from Elsevier for the 24th Ed. of the anatomy atlas "Sobotta" and the 'Sobotta Textbook of Anatomy' 2nd Ed.

Ethical approval and consent to participate The cadavers belonged to the Department of Anatomical Dissection and Donation, Medical University of Lodz.

Open Access This article is licensed under a Creative Commons Attribution 4.0 International License, which permits use, sharing, adaptation, distribution and reproduction in any medium or format, as long as you give appropriate credit to the original author(s) and the source, provide a link to the Creative Commons licence, and indicate if changes were made. The images or other third party material in this article are included in the article's Creative Commons licence, unless indicated otherwise in a credit line to the material. If material is not included in the article's Creative Commons licence and your intended use is not permitted by statutory regulation or exceeds the permitted use, you will need to obtain permission directly from the copyright holder. To view a copy of this licence, visit http://creativecommons.org/licenses/by/4.0/.

\section{References}

1. Moore KL, Dalley AF (1999) Clinical oriented anatomy. Lippincott Williams \& Wilkins, Philadeplphia

2. El-Naggar MM (2001) A study on the morphology of the coracobrachialis muscle and its relationship with the musculocutaneous nerve. Folia Morphol (Warsz) 60(3):217-224

3. El-Naggar MM, Al-Saggaf S (2004) Variant of the coracobrachialis muscle with a tunnel for the median nerve and brachial artery. Clin Anat 17(2):139-143. https://doi.org/10.1002/ca.10213

4. El-Naggar MM, Zahir FI (2001) Two bellies of the coracobrachialis muscle associated with a third head of the biceps brachii muscle. Clin Anat 14(5):379-382. https://doi.org/10.1002/ca.1067

5. Garbelotti SA et al (2017) An unusual case of accessory head of coracobrachialis muscle involving lateral cord of brachial plexus and its clinical significance. Folia Morphol 76(4):762-765. https ://doi.org/10.5603/FM.a2017.0033

6. Kopuz C et al (2003) A rare accessory coracobrachialis muscle: A review of the literature. Surg Radiol Anat 24(6):406-410. https ://doi.org/10.1007/s00276-002-0079-5

7. Loukas M, Aqueelah H (2005) Musculocutaneous and median nerve connections within, proximal and distal to the coracobrachialis muscle. Folia Morphol (Warsz) 64(2):101-108

8. Tatar I et al (2004) Innervation of the coracobrachialis muscle by a branch from the lateral root of the median nerve. Folia Morphol (Warsz) 63(4):503-506

9. Hayashi $\mathrm{M}$ et al (2017) A novel classification of musculocutaneous nerve variations: the relationship between the communicating branch and transposed innervation of the brachial flexors to the median nerve. Ann Anat 209:45-50. https://doi.org/10.1016/j. aanat.2016.08.004 
10. Ilayperuma I et al (2016) Coracobrachialis muscle: morphology, morphometry and gender differences. Surg Radiol Anat 38(3):335-340. https://doi.org/10.1007/s00276-015-1564-y

11. Bauones S, Moraux A (2015) The accessory coracobrachialis muscle: ultrasound and MR features. Skeletal Radiol 44(9):12731278. https://doi.org/10.1007/s00256-015-2153-1

12. Georgiev GP et al (2017) A novel type of coracobrachialis muscle variation and a proposed new classification. Cureus 9(7):1-6. https://doi.org/10.7759/cureus.1466

13. Georgiev GP et al (2018) Coracobrachialis longus muscle: humeroepitrochlearis. Cureus 10(5):5-9. https://doi.org/10.7759/cureu s. 2615

14. Mestdagh $\mathrm{H}$ et al (2002) Accessory coracobrachialis muscle as a cause of anterior impingement syndrome of the rotator cuff in an athlete. Eur J Orthop Surg Traumatol 12(2):96-98. https://doi. org/10.1007/s00590-002-0021-x

15. Olewnik $€$ et al (2018) Anatomical variations of the pronator teres muscle in a Central European population and its clinical significance. Anat Sci Int 93(2):299-306. https://doi.org/10.1007/s1256 5-017-0413-y

16. Olewnik $€$ et al (2020) Potential compression of the musculocutaneous, median and ulnar nerves by a very rare variant of the coracobrachialis longus muscle. Folia Morphol (Warsz). https:// doi.org/10.5603/fm.a2020.0085

17. Podgórski M et al (2019) 'superior biceps aponeurosis'-Morphological characteristics of the origin of the short head of the biceps brachii muscle. Ann Anat 223:85-89. https://doi.org/10.1016/j. aanat.2019.01.014

18. Podgórski MT et al (2019) Rotator cable in pathological shoulders: comparison with normal anatomy in a cadaveric study. Anat Sci Int 94(1):53-57. https://doi.org/10.1007/s12565-018-0447-9

19. Zielinska $\mathrm{N}$ et al (2020) A very rare case of an accessory subscapularis muscle and its potential clinical significance. Surg Radiol Anat. https://doi.org/10.1007/s00276-020-02531-6

20. Bardeen C (1906) Development and variation of the musculature of the inferior extremity and the neighboring regions of the trunk in man. Am J Anat 6:259-390. https://doi.org/10.1002/aja.10000 60108

21. Bardeen C (1905) Studies of the development of the human skeleton. Am J Anat. 4:265-302

22. Wood J (1864) On some varieties in human myology. Proc R Soc London 13:299-303

23. Mori M (1964) Statistics on the musculature of the Japanese. Okajimas Folia Anat Jpn 40:195-300

24. Gruber, W.: Die Musculi subscapulares (major et minor) und die neuen supernumären Schultermuskel des Menschen. Máemoires de I'Acadáemie Impáeriale des Sciences de Saint Páetersbourg par Divers Savans et Lus dans ses Assembláees. , Sankt Petersburg (1859).

25. Olewnik $€$ et al (2020) The co-occurrence of a four headed coracobrachialis muscke, split coracoid process and tunnel for the median and musculocutaneous nerve: the potential clinical relevance of a very rare variation. Surg Radiol Anat. https://doi. org/10.1007/s00276-020-02580-x

26. Ishimi A (1950) Studies on the musculature of the upper extremities of the Japanese fetuses. Report $\mathrm{n}$ the muscles of the brachium. Igaku Kentyu. 20:766-778

27. Bassett RW et al (1990) Glenohumeral muscle force and moment mechanics in a position of shoulder instability. J Biomech 23(5):2-3. https://doi.org/10.1016/0021-9290(90)90295-E

28. Kawasaki $\mathrm{T}$ et al (2014) Incidence of and risk factors for traumatic anterior shoulder dislocation: an epidemiologic study in highschool rugby players. J Shoulder Elb Surg 23(11):1624-1630. https://doi.org/10.1016/j.jse.2014.05.007
29. Pauly S et al (2013) Histopathologic evaluation of passive stabilizers in shoulder instability. J Shoulder Elb Surg 22(5):687-694. https://doi.org/10.1016/j.jse.2012.07.006

30. Walton $J$ et al (2002) The unstable shoulder in the adolescent athlete. Am J Sports Med 30(5):758-767. https://doi. org/10.1177/03635465020300052401

31. Saltzman BM et al (2015) Proximal coracobrachialis tendon rupture, subscapularis tendon rupture, and medial dislocation of the long head of the biceps tendon in an adult after traumatic anterior shoulder dislocation. Int J Shoulder Surg 9(2):52-55. https://doi. org/10.4103/0973-6042.154769

32. Olewnik L et al (2018) The plantaris muscle tendon and its relationship with the achilles tendinopathy. Biomed Res Int 2018:1-2. https://doi.org/10.1155/2018/9623579

33. Olewnik $€$ et al (2019) A cadaveric and sonographic study of the morphology of the tibialis anterior tendon-A proposal for a new classification. J Foot Ankle Res 12(1):1-8. https://doi. org/10.1186/s13047-019-0319-0

34. Olewnik $€$ (2019) A proposal for a new classification for the tendon of insertion of tibialis posterior. Clin Anat 32(4):557-565. https://doi.org/10.1002/ca.23350

35. Olewnik $€$ et al (2019) A proposal for a new classification of the fibular (lateral) collateral ligament based on morphological variations. Ann Anat 222:1-11. https://doi.org/10.1016/j.aanat .2018.10.009

36. Olewnik $€$ et al (2017) Anatomical variations of the palmaris longus muscle including its relation to the median nerve-a proposal for a new classification. BMC Musculoskelet Disord 18(1):1-9. https://doi.org/10.1186/s12891-017-1901-x

37. Olewnik $€$ (2019) Is there a relationship between the occurrence of frenular ligaments and the type of fibularis longus tendon insertion? Ann Anat 224:47-53. https://doi.org/10.1016/j.aanat .2019.03.002

38. Olewnik $€$ et al (2020) Proposal for a new classification of plantaris muscle origin and its potential effect on the knee joint. Ann Anat Anat Anzeiger. https://doi.org/10.1016/j.anat.2020.151506

39. Olewnik $€$ et al (2018) The plantaris muscle-rare relations to the neurovascular bundle in the popliteal fossa. The plantaris musclerare relations to the neurovascular bundle in the popliteal fossa. Folia Morphol (Warsz). https://doi.org/10.5603/FM.a2018.0039

40. Flatow EL et al (1989) An anatomic study of the musculocutaneous nerve and its relationship to the coracoid process. Clin Orthop Relat Res 244:166-171. https://doi.org/10.1097/00003086-19890 7000-00014

41. Remerand $\mathrm{F}$ et al (2010) Is the musculocutaneous nerve really in the coracobrachialis muscle when performing an axillary block? An ultrasound study. Anesth Analg 110(6):1729-1734. https:// doi.org/10.1213/ANE.0b013e3181dc25c8

42. Pećina M, Bojanić I (1993) Musculocutaneous nerve entrapment in the upper arm. Int Orthop 17(4):232-234. https://doi. org/10.1007/BF00194185

43. Venieratos D, Anagnostopoulou S (1998) Classification of communications between the musculocutaneous and median nerves. Clin Anat 11(5):327-331. https://doi.org/10.1002/(SICI)10982353(1998)11:5\%3c327::AID-CA6\%3e3.0.CO;2-M

44. Koizumi M (1989) A morphological study on the coracobrachialis muscle. Acta Anat Nippon 64(1):18-35

Publisher's Note Springer Nature remains neutral with regard to jurisdictional claims in published maps and institutional affiliations. 